\title{
SOLUTIONS OF NONSTANDARD INITIAL VALUE PROBLEMS FOR A FIRST ORDER ORDINARY DIFFERENTIAL EQUATION*
}

\author{
M. Venkatesulu and P.D.N. Srinivas \\ Department of Mathematics \\ Sri Sathya Sai Institute of Higher Learning \\ Prasanthinilayam - 515 134, A.P. \\ India
}

\begin{abstract}
Differential equations of the form $y^{\prime}=f\left(t, y, y^{\prime}\right)$, where $f$ is not necessarily linear in its arguments, represent certain physical phenomena and have been known to mathematicians for quite a long time. But a fairly general existence theory for solutions of the above type of problems does not exist because the (nonstandard) initial value problem $y^{\prime}=f\left(t, y, y^{\prime}\right), y\left(t_{0}\right)=y_{0}$ does not permit an equivalent integral equation of the conventional form. Hence, our aim here is to present a systematic study of solutions of the NSTD IVPS mentioned above.
\end{abstract}

First, we establish the equivalence of the NSTD IVP with a functional equation and prove the local existence of a unique solution of the NSTD IVP via the functional equation. Secondly, we prove the continuous dependence of the solutions on initial conditions and parameters. Finally, we prove a global existence result and present an example to illustrate the theory.

Key words: Nonstandard Initial Value Problems, existence, uniqueness, solution, scalar-valued connected continuous dependence, initial conditions, parameters, differentiable functional equation, Gronwall's inequality, contraction mapping theorem, fundamental theorem of integral calculus.

1980 AMS Subject Classification Codes: 34-XX, 34Axx, 34A15

\section{INTRODUCTION}

Differential equations of the form $y^{\prime}=f\left(t, y, y^{\prime}\right)$ where $f$ is not necessarily linear in its arguments represent certain physical phenomena and have been known by mathematicians for quite a long time. The well-known Clairut's and Chrystal's equations fall into this category [3]. A few authors, notably E.L. Ince [4], H.T. Davis [3] et.al., have given some methods for finding solutions of equations of the above type. In fact, these methods are best described as follows.

\footnotetext{
* Reccived April 21, 1989; Revised July 1, 1989
} 
If there exists $\left(t_{0}, y_{0}\right)$ such that the equation $y^{\prime}=f\left(t, y, y^{\prime}\right)$ can be solved for $y^{\prime}$ as a single-valued function of $(t, y)$ in a neighborhood of $\left(t_{0}, y_{0}\right)$, say $y^{\prime}=g(t, y)$, then the solution of the initial value problem (IVP) $y^{\prime}=g(t, y), y\left(t_{0}\right)=y_{0}$, if it exists, is also a solution of the original equation $y^{\prime}=f\left(t, y, y^{\prime}\right)$ (and satisfies the initial condition $\left.y\left(t_{0}\right)=y_{0}\right)$. Or, if there exists $\left(t_{0}, y_{0}\right)$, such that the equation $y^{\prime}=f\left(t, y, y^{\prime}\right)$ can be solved for $y^{\prime}$ as a multi-valued function of $(t, y)$ in a neighborhood of $\left(t_{0}, y_{0}\right)$, then a (nonunique) solution of the IVP $y^{\prime}=f\left(t, y, y^{\prime}\right), y\left(t_{0}\right)=y_{0}$ is given by a certain (not necessarily convergent) infinite series. For other cases, to the authors' knowledge, there does not seem to be any method for proving the existence of a solution of the above problem.

One obvious reason why there does not exist a fairly general existence theory for solutions of equations of the above type is that the nonstandard IVP $y^{\prime}=f\left(t, y, y^{\prime}\right)$, $y\left(t_{0}\right)=y_{0}$ does not permit an equivalent integral equation representation, whereas the IVP $y^{\prime}=g(t, y), y\left(t_{0}\right)=y_{0}$ does so. Hence our aim here is to present a systematic study of solutions of the nonstandard IVP mentioned above. First, we shall establish the equivalence of the nonstandard IVP with a functional equation (not the conventional integral equation), and shall prove the local existence of a unique solution of the nonstandard IVP via the functional equation. Secondly, we shall prove the continuous dependence of the solution on initial conditions, and parameters. Also, we shall establish a global existence result. Finally, we shall present an example to illustrate the theory.

Before indicating the section-wise split-up of the work, we shall introduce some notation and make some definitions. Let $R$ denote the real line, and let $C^{2}$ denote the twodimensional complex plane. Let $R \times C^{2}$ denote the cartesian product space of $R$ and $C^{2}$, taken in that order, equipped with the usual product topology. Let $D$ be a connected subset of $R \times C^{2}$ such that the interior of $D$, denoted by $D^{0}$, is nonempty. For a scalar (real or complex) valued function $y$ defined on an interval $I$ of $R$, let $y^{\prime}$ denote the derivative of $y$, if it exists. Let $f(t, y, z)$ be a scalar valued, not necessarily linear, function defined for $(t, y, z) \in D$.

Consider the nonstandard initial value problem (NSTD IVP)

$$
y^{\prime}=f\left(t, y, y^{\prime}\right), y\left(t_{0}\right)=y_{0}
$$

where $\left(t_{0}, y_{0}\right) \in D^{0} \cap$ (ty-plane).

Definition 1: By a solution y of the NSTD IVP (1), we mean a continuously differentiable scalar valued function $y(t)$ defined for $t \in I$, where I is some interval of the real line containing the point ' $t_{0}$ ' such that

i) $y\left(t_{0}\right)=y_{0}$,

ii) the triplet $\left(t, y(t), y^{\prime}(t)\right) \in D$ for all $t \in I$, and

iii) $y^{\prime}(t)=f\left(t, y(t), y^{\prime}(t)\right)$ holds good for all $t \in I$. 
Also, consider the (not necessarily linear) functional equation

$$
z(.)=f\left(., y_{0}+\int_{t_{0}}^{\dot{s}} z(s) d s, z(.)\right)
$$

Definition 2: By a solution $z$ of equation (2), we mean a continuous scalar valued function $z(t)$ defined for $t \in I$, where $I$ is some interval of the real line containing the point ' $t_{0}$ ' such that

$$
\begin{aligned}
& \text { ii) the triplet }\left(t, y_{0} .+\int_{t_{0}}^{t} z(s) d s, z(t)\right) \in D \text { for all } t \in D \text {, and } \\
& \text { iii) } z(t)=f\left(t, y_{0}+\int_{t_{0}}^{t} z(s) d s, z(t)\right) \text { holds good for all } t \in I \text {. }
\end{aligned}
$$

In section 1, we shall show that the NSTD IVP (1) and equation (2) are equivalent in the sense that IVP (1) has a (unique) solution if and only if equation (2) has a (unique) solution. Also, under usual hypotheses on $f$, we shall prove, using the contraction mapping theorem, that equation (2) has a unique local solution which, by the equivalence property, implies that IVP (1) has a unique local solution.

In section 2, we shall prove the continuous dependence of the solution of IVP (1) on initial conditions $\left(t_{0}, y_{0}\right)$, and on functions $f\left(t, y, y^{\prime}\right)$. Also, under continuity, uniform boundedness and Lipschitz conditions on $f$ (for $f$ defined on $R \times C^{2}$ ), we shall establish a global existence result for the solution of IVP (1).

In section 3 , by way of illustration, we shall prove the local existence of a unique solution of a concrete NSTD IVP.

\section{EQUIVALENCE OF THE NONSTANDARD IVP (1) AND FUNCTIONAL EQUATION (2), AND THE LOCAL EXISTENCE AND UNIQUENESS THEOREM}

The following lemmas establish the equivalence of the NSTD IVP (1) and functional equation (2).

Lemma 1: The nonstandard IVP (1) has a solution if and only if equation (2) has a solution.

Proof: Suppose that IVP (1) has a solution $y$. Then by definition 1, there exists an interval $I$ containing the point ' $t_{0}$ ' such that $y(t)$ is continuously differentiable (scalar-valued function) for $t \in I, y\left(t_{0}\right)=y_{0},\left(t, y(t), y^{\prime}(t)\right) \in D$ for all $t \in I$, and such that $y^{\prime}(t)=f\left(t, y(t), y^{\prime}(t)\right)$ holds good for all $t \in I$. 
Define $z(t)=y^{\prime}(t)$, for $t \in I$. Then, clearly, $z(t)$ is a continuous scalar-valued function defined for $t \in I, t_{0} \in I,\left(t, y_{0}+\int_{t_{0}}^{t} z(s) d s, z(t)\right)=\left(t, y(t), y^{\prime}(t)\right) \in D$ for all $t$ $\in I$, and $z(t)=f\left(t, y_{0}+\int_{t_{0}}^{t} z(s) d s, z(t)\right)$ holds good for all $t \in I$. Therefore, by definition $2, z(t)$ is a solution of equation (2).

Conversely, suppose that equation (2) has a solution $z$. Then, by definition 2 , there exists an interval $I$ containing ' $t_{0}$ ' such that $z(t)$ is a continuous scalar-valued function defined for $t \in I, \quad\left(t, y_{0}+\int_{t_{0}}^{t} z(s) d s, z(t)\right) \in D$ and that $z(t)=f\left(t, y_{0}+\int_{t_{0}}^{t} z(s) d s, z(t)\right)$ holds good for all $t \in \mathrm{I}$.

Define $y(t)=y_{0}+\int_{t_{0}}^{t} z(s) d s$, for $t \in I$. By the fundamental theorem of integral calculus [1], $y(t)$ is continuously differentiable for $t \in I, t_{0} \in I$, and $y^{\prime}(t)=z(t)$ for all $t$ $\in I$. Therefore, we have that $y\left(t_{0}\right)=y_{0}, .\left(t, y(t), y^{\prime}(t)\right)=\left(t, y_{0} .+\int_{t_{0}}^{t} z(s) d s, z(t)\right) \in D$ for all $t \in I$, and $y^{\prime}(t)=f\left(t, y(t), y^{\prime}(t)\right)$ holds good for all $t \in I$. Hence, by definition $1, y$ is a solution of IVP (1). This completes the proof.

Lemma 2: The nonstandard IVP (1) has a unique solution if and only if equation (2) has a unique solution.

Proof: Equivalently, we shall show that IVP (1) has more than one solution if and only if equation (2) has more than one solution.

Suppose that IVP (1) has two distinct solutions, say $y_{1} \neq y_{2}$ existing on a common interval $I$ containing ' $t_{0}$ '. Define $z_{i}(t)=y_{i}^{\prime}, t \in I, i=1,2$. We claim $z_{1} \not \equiv z_{2}$. For, if possible, let $z_{1}(t)=z_{2}(t)$ for all $t \in I$. Then $y_{1}^{\prime}(t)-y_{2}^{\prime}(t)=0$ for all $t \in I$, which upon integration implies that $y_{1}(t)-y_{2}(t)=k$, a constant, for all $t \in I$, and taking $t=t_{0}$, we get that $y_{1}\left(t_{0}\right)-y_{2}\left(t_{0}\right)=y_{0}-y_{0}=0=k$. Hence $y_{1}(t)=y_{2}(t)$ for all $t \in I$, which is a contradiction. Therefore, $z_{1} \neq z_{2}$ and by lemma 1 we get that $z_{1}$ and $z_{2}$ are two distinct solutions of equation (2).

Conversely, suppose that $z_{1} \neq z_{2}$ are two distinct solutions of equation (2) existing on a common interval containing ' $t_{0}$ '. Define

$$
y_{i}(t)=y_{0}+\int_{i}^{t_{0}} z_{i}(s) d s, i=1,2 .
$$

We claim that $y_{1} \not \equiv y_{2}$. For, if possible, let $y_{1}(t)=y_{2}(t)$ for all $t \in I$. Then by the fundamental theorem of integral calculus we get that $z_{1}(t)=z_{2}(t)$ for all $t \in I$, which is a 
contradiction. Therefore $y_{1} \not \equiv y_{2}$ and by lemma 1 we get that $y_{1}$ and $y_{2}$ are two distinct solutions of IVP (1). This completes the proof.

The following corollary follows immediately from lemmas 1 and 2 .

Corollary 1: The nonstandard IVP (1) has a unique solution if and only if equation (2) has a unique solution.

Next, we shall prove a local existence and uniqueness theorem for the solution of IVP (1).

Let $D$ be a connected subset of $R \times C^{2}$ defined by

$$
D=\left\{(t, y, z) \in R \times C^{2}|| t-t_{0}|\leq a,| y-y_{0}|\leq b,| z \mid \leq c\right\}
$$

where $a, b$ and $c$ are positive constants. We note that $D^{0}$ is nonempty and $\left(t_{0}, y_{0}\right) \in D^{0} \cap$ (ty-plane). Let $f$ be a scalar valued function defined on $D$ satisfying the following conditions:

i) $f$ is continuous with respect to $(t, y, z) \in D$,

ii) $|f(t, y, z)| \leq c$ for all $(t, y, z) \in D$, and

iii) $\left|f\left(t, y_{1}, z_{1}\right)-f\left(t, y_{2}, z_{2}\right)\right| \leq k_{1}\left|y_{1}-y_{2}\right|+k_{2}\left|z_{1}-z_{2}\right|$ for all $\left(t, y_{1}, z_{1}\right),\left(t, y_{2}, z_{2}\right) \in D$, where $k_{1}>0$ and $0 \leq k_{2}<1$ are constants.

Theorem 1 (Local existence and uniqueness theorem): Under conditions (i)-(iii), the nonstandard IVP (1) has a unique solution existing on the interval $\left[t_{0}-\alpha, t_{0}+\alpha\right]$, where $\alpha$ is a real number such that

$$
0<\alpha<\min \left(\frac{1-k_{2}}{k_{1}}, b / c, a\right) .
$$

Proof: By corollary 1, it is enough to prove the existence of a unique solution $z$ of equation (2) on the interval $\left[t_{0}-\alpha, t_{0}+\alpha\right]$, which we shall accomplish by making use of contraction mapping theorem in a suitable function space.

To this end, let $I=\left[t_{0}-\alpha, t_{0}+\alpha\right]$ where $\alpha$ is a real number satisfying relation (3). Consider the Banach space $C(I)$ of all continuous scalar valued functions defined on $I$, equipped with the supremum norm given by $\|y\|=\sup _{t \in}|y(t)|, y \in C(I)$. Let $M=\{z \in$ $C(I) \mid\|z\| \leq c\}$. Clearly, $M$ is a nonempty closed subset of $C(I)$. For $z \in M$, we have $|z(t)| \leq c$ for all $t \in I$, and letting

$$
y(t)=y_{0}+\int_{t_{0}}^{t} z(s) d s, t \in I
$$

we get that $\left|y(t)-y_{0}\right| \leq\left|\int_{t_{0}}^{t}\right| z(s)|d s|$ (by (3)).

Thus for every $t \in I$, the triplet $(t, y(t), z(t)) \in D$. Now define a map $F: M \rightarrow C(I)$ by

$$
(F z)(t)=f\left(t, y_{0}+\int_{t_{0}}^{t} z(s) d s, z(t)\right), t \in I
$$


From conditions ( $i$ ) and (ii), it easily follows that $F$ indeed maps $M$ into itself. To verify that $F$ is a contraction on $M$, let $z_{1}$ and $z_{2} \in M$, and consider

$$
\begin{aligned}
\left\|F z_{1}-F z_{2}\right\| & =\sup _{t} f\left(t, y_{0}+\int_{t_{0}}^{t} z_{1}(s) d s, z_{1}(t)\right)-f\left(t, y_{0}+\int_{t_{0}}^{t} z_{2}(s) d s, z_{2}(t)\right) \\
& \leq \sup _{t}\left\{\left(k_{1} \mid\left(\int_{t 0}^{t}\left|z_{1}(s)-z_{2}(s)\right| d s\left|+k_{2}\right| z_{1}(t)-z_{2}(t) \mid\right\}\right.\right. \text { (by (iii)) } \\
& \leq \mathrm{k} 1 \sup _{t \in P} \mid\left(\int_{t 0}^{t}\left\|z_{1}-z_{2}\right\| d s \mid+k_{2}\left\|z_{1}-z_{2}\right\|\right. \\
& \leq\left(k_{1} \alpha+k_{2}\right)\left\|z_{1}-z_{2}\right\|
\end{aligned}
$$

By relation (3), $0 \leq k_{1} \alpha+k_{2}<1$, and hence $F$ is a contraction map on $M$.

Consequently, by contraction mapping theorem [5], $F$ has a unique fixed point (in $M)$. That is, there exists a unique $z \in M$ such that

$$
z(t)=\left(F_{z}\right)(t)=f\left(t, y_{0}+\int_{t_{0}}^{t} z(s) d s, z(t)\right) \text { for all } t \in I .
$$

This completes the proof.

Corollary 2: Under the hypotheses of theorem 1, IVP (1) has a unique solution existing on the interval $\left(t_{0}-\beta, t_{0}+\beta\right)$, where $\beta=\min \left(\frac{1-k_{2}}{k_{1}}, b / c, a\right)$.

Proof follows immediately from theorem 1 , since the unique solution of IVP (1) exists on the interval $\left[t_{0}-\alpha, t_{0}+\alpha\right]$ for every $\alpha$ such that $0<\alpha<\beta$.

\section{CONTINUOUS DEPENDENCE OF THE SOLUTION OF IVP (1) ON INITIAL CONDITIONS, PARAMETERS, AND THE GLOBAL EXISTENCE THEOREM}

Theorem 2 (Continuous dependence of the solution on initial conditions): Let the hypotheses of the theorem be true. Let $t_{0}^{\prime} \in\left(t_{0}-\beta, t_{0}+\beta\right)$, where $\beta=\min \left(\frac{1-k_{2}}{k_{1}}, b / c, a\right.$, and let $\left|y_{0}-y_{0}^{\prime}\right|<b$. Let $\tilde{D}=\left\{(t, y, z) \in \boldsymbol{R} \times \mathbb{C}^{2}|| t-t_{0}^{\prime}|\leq a| y-,y_{0}^{\prime}|\leq b| z \mid, \leq c\right\}$. Suppose that $f$ is also defined on $\tilde{D}$ and satisfies conditions $(i)-(i i i)$ on $\tilde{D}$. Let $y_{1}$ be the unique solution of the IVP $y^{\prime}=f(t, y, y), y\left(t_{0}\right)=y_{0}$ existing on the interval $(t 0-\beta, t 0+\beta)$. Let $y_{2}$ be the unique solution of the IVP $y^{\prime}=f(t, y, y), y\left(t_{0}^{\prime}\right)=y_{0}^{\prime}$ existing on the interval $\left(t_{0}^{\prime}-\beta, t_{0}^{\prime}+\beta\right)$. Let $r_{1}=\max \left(t_{0}-\beta, t_{0}^{\prime}-\beta\right)$ and $r_{2}=\min \left(t_{0}+\beta, t_{0}^{\prime}+\beta\right)$. Clearly $t_{0}$ and $t_{0}^{\prime} \in\left(r_{1}, r_{2}\right)$, and both $y_{1}$ and $y_{2}$ exist on the interval $\left(r_{1}, r_{2}\right)$. Suppose that $\left|t_{0}-t_{0}^{\prime}\right|<\delta$ and $\left|y_{0}-y_{0}^{\prime}\right|<\delta$ where $0 \leq \delta<\left(r_{2}-r_{1}\right)$, then 


$$
\left|y_{1}(t)-y_{2}(t)\right| \leq\left(1+c+\frac{k_{1}\left(r_{2}-r_{1}\right)(1+c)}{1-k_{2}} e^{\left(k_{1}\left(r_{2}-r_{1}\right) /\left(1-k_{2}\right)\right)}\right) \delta
$$

for all $t \in\left(r_{1}, r_{2}\right)$.

Proof: Let $\phi_{1}(t)=y_{1}^{\prime}(t)$ and $\phi_{2}(t)=y_{2}^{\prime}(t)$. Then

$$
\phi_{1}(t)=f\left(t, y_{0}+\int_{t_{0}}^{t} \phi_{1}(s) d s, \phi_{1}(t)\right), \text { and } \phi_{2}(t)=f\left(t, y_{0}^{\prime}+\int_{t_{0}^{\prime}}^{t} \phi_{2}(s) d s, \phi_{2}(t)\right)
$$

for all $t \in\left(r_{1}, r_{2}\right)$.

Therefore, for $t \in\left(r_{1}, r_{2}\right)$, we have

$$
\begin{aligned}
& \left|\phi_{1}(t)-\phi_{2}(t)\right|=\left|f\left(t, y_{0}+\int_{t_{0}}^{t} \phi_{1}(s) d s, \phi_{1}(t)\right)-f\left(t, y_{0}^{\prime}+\int_{t_{0}^{\prime}}^{t} \phi_{2}(s) d s, \phi_{2}(t)\right)\right| \\
& \quad \leq k_{1}\left|y_{0}-y_{0}^{\prime}+\int_{t_{0}}^{t} \phi_{1}(s) d s-\int_{t_{0}^{\prime}}^{t} \phi_{2}(s) d s\right|+k_{2}\left|\phi_{1}(t)-\phi_{2}(t)\right|(\text { by }(i i i)) \\
& (4) \quad \leq k_{1}\left|y_{0}-y_{0}^{\prime}\right|+k_{1}\left|\int_{t_{0}}^{t_{0}^{\prime}} \phi_{1}(s) d s\right|+k_{1}\left|\int_{t_{0}^{\prime}}^{t}\left(\phi_{1}(s)-\phi_{2}(s)\right) d s\right|+k_{2}\left|\phi_{1}(t)-\phi_{2}(t)\right| \\
& \quad \leq k_{1} \delta+k_{1}\left|\int_{t_{0}}^{t_{0}^{\prime}}\right| \phi_{1}(s)|d s|+k_{1}\left|\int_{t_{0}^{\prime}}^{t}\right| \phi_{1}(s)-\phi_{2}(s)|d s|+k_{2}\left|\phi_{1}(t)-\phi_{2}(t)\right| \\
& \leq k_{1} \delta+k_{1} c \delta+k_{1}\left|\int_{t_{0}^{\prime}}^{t}\right| \phi_{1}(s)-\phi_{2}(s)|d s|+k_{2}\left|\phi_{1}(t)-\phi_{2}(t)\right|
\end{aligned}
$$

which implies that

$$
\left|\phi_{1}(t)-\phi_{2}(t)\right| \leq \frac{k_{1}(1+c) \delta}{1-k_{2}}+\frac{k_{1}}{1-k_{2}} \mid\left(\int_{t}^{t}\left|\phi_{1}(s)-\phi_{2}(s)\right| d s \mid .\right.
$$

Now, by Gronwall's inequality [2], we get from the above that

$$
\begin{aligned}
\left|\phi_{1}(t)-\phi_{2}(t)\right| & \leq\left(\frac{(1+c) k_{1} \delta}{1-k_{2}}\right) e^{k_{1} /\left(1-k_{2}\right)\left|t-t_{0}^{\prime}\right|} \\
& \leq\left(\frac{(1+c) k_{1} \delta}{1-k_{2}}\right) e^{k_{1}\left(r_{1}-r_{2}\right) /\left(1-k_{2}\right)}
\end{aligned}
$$


for all $t \in\left(r_{1}, r_{2}\right)$.

Consequently, for $t \in\left(r_{1}, r_{2}\right)$, we get that

$$
\begin{aligned}
\left|y_{1}(t)-y_{2}(t)\right| & =\left|y_{0}+\int_{t_{0}}^{t} \phi_{1}(s) d s-y_{0}^{\prime}-\int_{t_{0}^{\prime}}^{t} \phi_{2}(s) d s\right| \\
& \leq\left|y_{0}-y_{0}^{\prime}\right|+\left|\int_{t_{0}}^{t_{0}^{\prime}}\right| \phi_{1}(s)|d s|+\left|\int_{t_{0}^{\prime}}^{t}\right| \phi_{1}(s)-\phi_{2}(s)|d s| \\
& \leq \delta+c \delta+\left(\frac{(1+c) k_{1} \delta}{1-k_{2}}\right)\left(r_{2}-r_{1}\right) e^{k_{1}\left(r_{2}-r_{1}\right) /\left(1-k_{2}\right)}
\end{aligned}
$$

This completes the proof.

Corollary 3: Under the hypotheses of theorem 2, if $t, t^{\prime} \in\left(r_{1}, r_{2}\right)$ and $\left|t-t^{\prime}\right|<\delta$, then

$$
\left|y_{1}(t)-y_{2}(t)\right| \leq\left(1+2 c+\left(\frac{k_{1}(1+c)\left(r_{2}-r_{1}\right)}{1-k_{2}}\right) e^{k_{1}\left(r_{2}-r_{1}\right) /\left(1-k_{2}\right)}\right) \delta \text {. }
$$

Proof: For $t, t^{\prime} \in\left(r_{1}, r_{2}\right)$ and $\mid t-t^{\prime}<\delta$, we have

$$
\begin{aligned}
\left|y_{1}(t)-y_{2}\left(t^{\prime}\right)\right| & \leq\left|y_{1}(t)-y_{2}(t)\right|+\left|y_{2}(t)-y_{2}\left(t^{\prime}\right)\right| \\
& \leq\left|y_{1}(t)-y_{2}(t)\right|+\left|y_{0}^{\prime}+\int_{t_{0}^{\prime}}^{t} \phi_{2}(s) d s-y_{0}^{\prime}-\int_{t_{0}^{\prime}}^{t^{\prime}} \phi_{2}(s) d s\right| \\
& \leq\left|y_{1}(t)-y_{2}(t)\right|+\left|\int_{t}^{t^{\prime}}\right| \phi_{2}(s)|d s| \\
& \leq\left|y_{1}(t)-y_{2}(t)\right|+c \delta \\
& \leq\left(1+c+\left(\frac{k_{1}(1+c)\left(r_{2}-r_{1}\right)}{1-k_{2}}\right) e^{\left.k_{1}\left(r_{2}-r_{1}\right) /\left(1-k_{2}\right)\right) \delta+c \delta}\right.
\end{aligned}
$$

(by theorem 2).

This completes the proof.

Theorem 3 (Continuous dependence of the solution on parameters): Let the hypotheses of theorem 1 be true. Let $y_{1}$ be the unique solution of the IVP $y^{\prime}=f(t, y, y), y\left(t_{0}\right)=y_{0}$ existing on the interval $\left(t_{0}-\beta, t_{0}+\beta\right)$, where $\beta=\min \left(\frac{1-k_{2}}{k_{1}}, b / c, a\right)$. Let $\left|t_{0}-t_{0}^{\prime}\right|<\beta$ and $\left|y_{0}-y_{0}^{\prime}\right|<\beta$. 
Let $\tilde{D}=\left\{(t, y, z) \in R \times \mathbb{C}|| t-t_{0}^{\prime}\left|\leq a_{1},\right| y-y_{0}^{\prime}\left|\leq b_{1},\right| z \mid \leq c_{1}\right\}$ where $a_{1}, b_{1}$ are positive constants and $0<c_{1} \leq c$. We note that $D \cap \tilde{D} \neq \phi$. Let $g(t, y, z)$ be a scalar valued function defined for $(t, y, z) \in \tilde{D}$ satisfying the following conditions:

i) $g(t, y, z)$ is continuous with respect to $(t, y, z) \in \tilde{D}$, ii) $|g(t, y, z)| \leq c_{1}$ for all $(t, y, z) \in \tilde{D}$,

iii) $\left|g\left(t, y_{1}, z_{1}\right)-g\left(t, y_{2}, z_{2}\right)\right| \leq k_{3}\left|y_{1}-y_{2}\right|+k_{4}\left|z_{1}-z_{2}\right|$, for all $\left(t, y_{1}, z_{1}\right),\left(t, y_{2}, z_{2}\right) \in \tilde{D}$ where $k_{3}>0$ and $0 \leq k_{4}<1$ are constants, and iv) $|f(t, y, z)-g(t, y, z)| \leq \theta$ for all $(t, y, z) \in D \cap \tilde{D}$ where $\theta \geq 0$ is a constant.

Let $y_{2}$ be the unique solution of the IVP $y^{\prime}=g(t, y, y), y\left(t_{0}^{\prime}\right)=y_{0}^{\prime}$ existing on the interval $\left(t_{0}^{\prime}-\tilde{\beta}, t_{0}^{\prime}+\tilde{\beta}\right)$ where $\tilde{\beta}=\min \left(\frac{1-k_{4}}{k_{3}}, b_{1} / c_{1}, a_{1}\right)$. Let $\eta=\min (\beta, \tilde{\beta})$. Futher, assume that $\left|t_{0}-t_{0}^{\prime}\right|<\eta$. Let $r_{1}=\max \left(t_{0}-\eta, t_{0}^{\prime}-\eta\right)$ and $r_{2}=\min \left(t_{0}+\eta, t_{0}^{\prime}+\eta\right)$. Clearly, $t_{0}$ and $t_{0}^{\prime} \in\left(r_{1}, r_{2}\right)$, and both $y_{1}$ and $y_{2}$ exist on the interval $\left(r_{1}, r_{2}\right)$. Now suppose that $\left|t_{0}-t_{0}^{\prime}\right|<\delta$ and $\left|y_{0}-y_{0}^{\prime}\right|<\delta$ where

$$
0 \leq \delta \leq \min \left(\eta, b-c\left(r_{2}-r_{1}\right)\right),
$$

then

$$
\left|y_{1}(t)-y_{2}(t)\right| \leq\left(1+c_{1}\right) \delta+\left(\frac{(1+c) k_{1} \delta+\theta}{1-k_{2}}\right)\left(r_{2}-r_{1}\right) e^{\left(\frac{k_{1}}{1-k_{2}}\left(r_{2}-r_{1}\right)\right)}
$$

for all $t \in\left(r_{1}, r_{2}\right)$.

Proof: Let $\phi_{1}=y_{1}^{\prime}$ and $\phi_{2}=y_{2}^{\prime}$. Then $\phi_{1}(t)=f\left(t, y_{0}+\int_{t_{0}}^{t} \phi_{1}(s) d s, \phi_{1}(t)\right)$ and $\phi_{2}(t)=g\left(t, y_{0}^{\prime}+\int_{t_{0}}^{t} \phi_{2}(s) d s, \phi_{2}(t)\right)$ for all $t \in\left(r_{1}, r_{2}\right)$. Now for $\left(r_{1}, r_{2}\right)$, we have that $\left|t-t_{0}\right| \leq a,\left|\phi_{2}(t)\right| \leq c_{1} \leq c$, and

$\left|y_{0}^{\prime}+\int_{t_{0}}^{\mathrm{t}} \phi_{2}(s) d s,-y_{0}\right| \leq\left|y_{0}-y_{0}^{\prime}\right|+\left|\int_{t_{0}}^{\mathrm{t}}\right| \phi_{2}(s)|d s| \leq \delta+c_{1}\left(r_{2}-r_{1}\right) \leq \delta+c\left(r_{2}-r_{1}\right) \leq b$

(by (5)).

Hence $\left(t, y_{0}^{\prime}+\int_{t_{0}}^{t} \phi_{2}(s) d s, \phi_{2}(t)\right) \in D$.

Therefore,

$\left|\phi_{1}(t)-\phi_{2}(t)\right|=\left|f\left(t, y_{0}+\int_{t_{0}}^{t} \phi_{1}(s) d s, \phi_{1}(t)\right)-g\left(t, y_{0}^{\prime}+\int_{t_{0}}^{t} \phi_{2}(s) d s, \phi_{2}(t)\right)\right|$ 


$$
\begin{aligned}
& \leq\left|f\left(t, y_{0}+\int_{t_{0}}^{t} \phi_{1}(s) d s, \phi_{1}(t)\right)-f\left(t, y_{0}^{\prime}+\int_{t_{0}}^{t} \phi_{2}(s) d s, \phi_{2}(t)\right)\right| \\
& \quad+\left|f\left(t, y_{0}^{\prime}+\int_{t_{0}}^{t} \phi_{2}(s) d s, \phi_{2}(t)\right)-g\left(t, y_{0}^{\prime}+\int_{t_{0}}^{t} \phi_{2}(s) d s, \phi_{2}(t)\right)\right| \\
& \leq(1+c) k_{1} \delta+k_{1}\left|\int_{t_{0}}^{t}\right| \phi_{1}(s)-\phi_{2}(s)|d s|+k_{2}\left|\phi_{1}(t)-\phi_{2}(t)\right|+\theta,
\end{aligned}
$$

(by (4) and (iv))

which implies that

$$
\left|\phi_{1}(t)-\phi_{2}(t)\right| \leq\left(\frac{(1+c) k_{1} \delta+\theta}{1-k_{2}}\right)+\left.\frac{k_{1}}{1-k_{2}}\right|_{t_{0}^{\prime}} ^{t}\left|\phi_{1}(s)-\phi_{2}(s)\right| d s \mid .
$$

Hence by Gronwall's inequality we get that

$$
\left|\phi_{1}(t)-\phi_{2}(t)\right| \leq\left(\frac{(1+c) k_{1} \delta+\theta}{1-k_{2}}\right) e^{\left(\frac{k_{1}}{1-k_{2}}\left(r_{2}-r_{1}\right)\right)}, \text { for all } t \in\left(r_{1}, r_{2}\right) \text {. }
$$

Consequently, for $t \in\left(r_{1}, r_{2}\right)$, we obtain that

$$
\begin{aligned}
\left|y_{1}(t)-y_{2}(t)\right|= & \left|y_{0}-y_{0}^{\prime}+\int_{t_{0}}^{t} \phi_{1}(s) d s-\int_{t_{0}^{\prime}}^{t} \phi_{2}(s) d s\right| \\
& \leq\left|y_{0}-y_{0}^{\prime}\right|+\left|\int_{t_{0}^{\prime}}^{t_{0}} \phi_{2}(s)\right| d s|+| \int_{t_{0}}^{t}\left|\phi_{1}(s)-\phi_{2}(s)\right| d s \mid \\
& \leq \delta+c_{1} \delta+\left(\frac{(1+c) k_{1} \delta+\theta}{1-k_{2}}\right)\left(r_{2}-r_{1}\right) e^{\left(\frac{k_{1}}{1-k_{2}}\left(r_{2}-r_{1}\right)\right)} .
\end{aligned}
$$

This completes the proof.

Theorem 4 (Global existence theorem) Let $f$ be a scalar valued function defined on the whole of $\boldsymbol{R} \times C^{2}$ such that $f$ satisfies conditions (i)-(iii) on $R \times C^{2}$. Then for every initial data $\left(t_{0}, y_{0}\right) \in \boldsymbol{R} \times C$, the IVP(1) has a unique solution existing on the entire real line $(-\infty,+\infty)$.

Proof: For the initial data $\left(t_{0}, y_{0}\right) \in R \times C$, consider the set

$$
D_{1}=\left\{(t, y, z) \in R \times C^{2}|| t-t_{0}|\leq a,| y-y_{0}|\leq b,| z \mid \leq c\right\}
$$

where $a, b$ are some positive constants, and $c$ is the bound for $f$ given by (ii). From the hypotheses of the theorem, it clearly follows that $f$ satisfies conditions (i) - (iii) on $D_{1}$. Hence, by theorem 1 , there exists a unique solution $y_{1}$ of the IVP(1) on the interval 
$\left[t_{0}-\alpha, t_{0}+\alpha\right]$, for every $\alpha$ such that $0<\alpha<\min \left(\frac{1-k_{2}}{k_{1}}, b / c, a\right)$. Now fix $\alpha$ and consider the initial data $\left(t_{0}+\alpha, y_{1}\left(t_{0}+\alpha\right)\right)$. Let

$$
D_{2}=\left\{(t, y, z) \in R \times C^{2}|| t-t_{0}-\alpha|\leq a,| y-y_{1}\left(t_{0}+\alpha\right)|\leq b,| z \mid \leq c\right\} .
$$

Again $f$ satisfies conditions ( $i$ ) - (iii) on $D_{2}$, and hence by theorem 1 , there exists a unique solution $y_{2}$ of the IVP

$$
y^{\prime}=f(t, y, y), y\left(t_{0}+\alpha\right)=y_{1}\left(t_{0}+\alpha\right),
$$

on the interval $\left[t_{0}, t_{0}+2 \alpha\right]$. Also, by the uniqueness of the solution, we have that $y_{1}(t)=y_{2}(t)$, for $t \in\left[t_{0}, t_{0}+\alpha\right]$. Now, define

$$
y(t)=\left\{\begin{array}{l}
y_{1}(t), \text { for } t \in\left[t_{0}-\alpha, t_{0}+\alpha\right], \\
y_{2}(t), \text { for } t \in\left[t_{0}+\alpha, t_{0}+2 \alpha\right]
\end{array} .\right.
$$

Clearly, $y(t)$ is a unique solution of the IVP(1) existing on the interval $\left[t_{0}-\alpha, t_{0}+2 \alpha\right]$.

Next take the new initial data $\left(t_{0}+2 \alpha, y_{2}\left(t_{0}+2 \alpha\right)\right)$ and consider the set

$$
D_{3}=\left\{(t, y, z) \in R \times C^{2}|| t-t_{0}-2 \alpha|\leq a,| y-y_{2}\left(t_{0}+2 \alpha\right)|\leq b,| z \mid \leq c\right\}
$$

As before, $f$ satisfies conditions ( $i$ ) - (iii) on $D_{3}$, and hence there exists a unique solution $y_{3}$ of IVP

$$
y^{\prime}=f\left(t, y, y^{\prime}\right), y\left(t_{0}+2 \alpha\right)=y_{2}\left(t_{0}+2 \alpha\right),
$$

on the interval $\left[t_{0}+\alpha, t_{0}+3 \alpha\right]$. Also, we have that $y_{2}(t)=y_{3}(t)$, for $t \in\left[t_{0}+\alpha, t_{0}+3 \alpha\right]$. Now, define

$$
y(t)=\left\{\begin{array}{l}
y_{1}(t), \text { for } t \in\left[t_{0}-\alpha, t_{0}+\alpha\right] \\
y_{2}(t), \text { for } t \in\left[t_{0}+\alpha, t_{0}+2 \alpha\right] \\
y_{3}(t), \text { for } t \in\left[t_{0}+2 \alpha, t_{0}+3 \alpha\right]
\end{array} .\right.
$$

Thus the IVP(1) has a unique solution $y$ existing on the interval $\left[t_{0}-\alpha, t_{0}+3 \alpha\right]$.

Proceeding in this way indefinitely, we see that the solution $y$ of the IVP(1) can be extended uniquely to the entire interval $\left[t_{0}-\alpha,+\infty\right)$. Similarly it can be shown that the solution $y$ can be extended uniquely to the interval $\left(-\infty, t_{0}-\alpha\right]$. This proves that the IVP(1) has a unique solution existing on the entire real line $(-\infty,+\infty)$. Hence the proof is complete.

\section{ILLUSTRATIVE EXAMPLE}

Consider the IVP

$$
y^{\prime}=\alpha e^{t}+\beta y+\not y_{2} \cos \left|y^{\prime}\right|, y(0)=1
$$


where $\alpha, \beta$, and $\gamma$ are (real or complex) constants. The problem that is of interest to us comes when $\gamma \neq 0$.

Below, we shall prove that IVP(6) has unique solution existing on $(-1,+1)$ for certain values of $\alpha, \beta$, and $\gamma$.

Theorem 5: Suppose that the constants $\alpha, \beta$, and $\gamma \neq 0$ satisfy the inequalities

$$
\begin{aligned}
& |\alpha| e+2|\beta|+4|\gamma| \leq 1, \\
& \text { and }|\beta|+8|\gamma| \leq 1 .
\end{aligned}
$$

Then the IVP(6) has a unique solution existing on the interval $(-1,1)$.

Proof: Let

$$
D=\left\{(t, y, z) \in R \times C^{2}|| t|\leq 1,| y-1|\leq 1,| z \mid \leq 1\right\} .
$$

Clearly, $D$ is a connected subset of $R \times C^{2}$, and $\left(t_{0}, y_{0}\right)=(0,1) \in D^{0} \cap(t y$-plane). Here, we have $f(t, y, z)=\alpha e^{t}+\beta y+\gamma y_{2} \cos |z|$ and is continuous for $(t, y, z) \in D$. Also, for $(t, y, z) \in D$, we have that

$$
\begin{aligned}
|f(t, y, z)| & \leq\left|\alpha\left\|e^{t}|+| \beta|| y|+| y\right\| y_{2} \| \cos \right| z|| \\
& \leq|\alpha \| e|+2|\beta|+4|y| \\
& \leq 1
\end{aligned}
$$

Now, for $\left(t, y_{1}, z_{1}\right)$ and $\left(t, y_{2}, z_{2}\right) \in D$, consider

$$
\begin{aligned}
\left|f\left(t, y_{1}, z_{1}\right)-f\left(t, y_{2}, z_{2}\right)\right|= & \left|\beta y_{1}-\beta y_{2}+\not y_{1}{ }^{2} \cos \right| z_{1}\left|-\not y_{2}{ }^{2} \cos \right| z_{2}|| \\
& \leq|\beta|\left|y_{1}-y_{2}\right|+|\gamma|\left|y_{1}{ }^{2} \cos \right| z_{1}\left|-y_{2}{ }^{2} \cos \right| z_{2}|| \\
& \leq|\beta|\left|y_{1}-y_{2}\right|+\mid y\left(\left(\left.\right|_{1}{ }^{2}-y_{2}{ }^{2}|| \cos \left|z_{1}\right|+\left|y_{2}{ }^{2}\right||\cos | z_{1}|-\cos | z_{2} \mid\right)\right. \\
& \leq|\beta|\left|y_{1}-y_{2}\right|+|\gamma|\left(\left(\left|y_{1}\right|+\left|y_{2}\right|\right)\left|y_{1}-y_{2}\right|\right. \\
& \left.\quad+\left|y_{2}\right| 22 \sin \left(\frac{\left|z_{1}\right|+\left|z_{2}\right|}{2}\right) \sin \left(\frac{\left|z_{1}\right|-\left|z_{2}\right|}{2}\right)\right) \\
& \leq|\beta|\left|y_{1}-y_{2}\right|+4|\gamma|\left|y_{1}-y_{2}\right|^{2}+4|\gamma| z_{1}-\left.z_{2}\right|^{2} \quad \text { (by (9)) } \\
& =k_{1}\left|y_{1}-y_{2}\right|+k_{2}\left|z_{1}-z_{2}\right|
\end{aligned}
$$

where $k_{1}=|\beta|+4|\not|$ and $k_{2}=4|\not|$. By inequality (8), we have that $k_{1}>0$ and $0<k_{2}<1$ (since $\gamma \neq 0$ ). Thus $f$ satisfies conditions $(i)$-(iii) on $D$, and hence by theorem 1 , the $\operatorname{IVP}(6)$ has a unique solution existing on the interval $(-\delta, \delta)$ where $\delta=\min \left(\frac{1-4|x|}{|\beta|+4|\gamma|}, 1\right)$. But by inequality ( 8 ), we have $\delta=1$. This completes the proof of the theorem.

Remark 1: Theorem 1 guarantees only the existence of unique solutions of nonstandard IVPs and does not provide methods of finding these solutions explicitly. Nevertheless, in a subsequent paper we shall present numercial methods for finding the (approximate) solutions of the nonstandard IVPs.

Remark 2: The theory developed here also holds good for nonstandard IVPs for 
( $m$-dimensional) vector differential equations and in this case we simply replace the (modulus) $1 . \mathrm{l}$ by the $m$-dimensional Euclidean norm $1.1 \mathrm{~m}$.

\section{ACKNOWLEDGEMENT}

The authors dedicate the work to the chancellor of the institute Bhagawan Sri Sathya Sai Baba.

\section{REFERENCES}

[1] Bartle, R.G., The Elements of Real Analysis, John Wiley, New York, 1976.

[2] Brauer, F., and J.A. Nohel, The Qualitative Theory of Ordinary Differential Equations-An Introduction, W.A. Benjamin, New York, 1969.

[3] Davis, H.T., Introduction to Nonlinear Differential and Integral Equations, Dover Publications, New York, 1962.

[4] Ince, E.L., Ordinary Differential Equations, Dover publications, New York, 1956.

[5] Krasnoselskii, M.A., et.al, Approximate Solution of Operator Equations, WoltersNoordhoff Publishing, Groningen, 1972. 


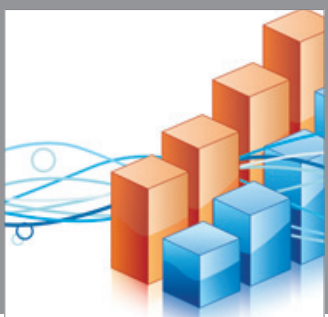

Advances in

Operations Research

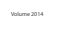

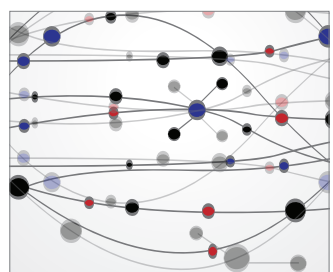

\section{The Scientific} World Journal
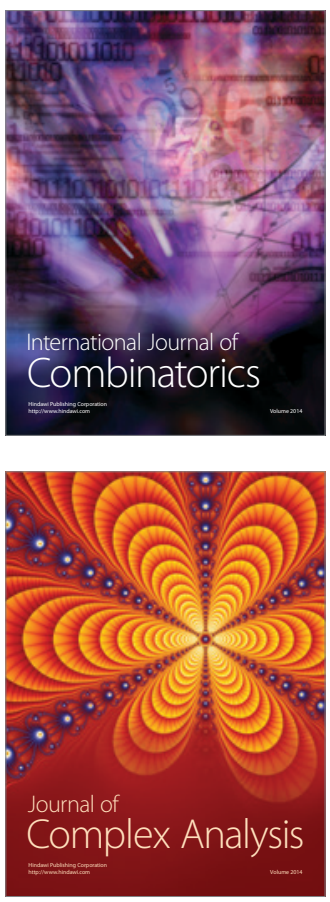

International Journal of

Mathematics and

Mathematical

Sciences
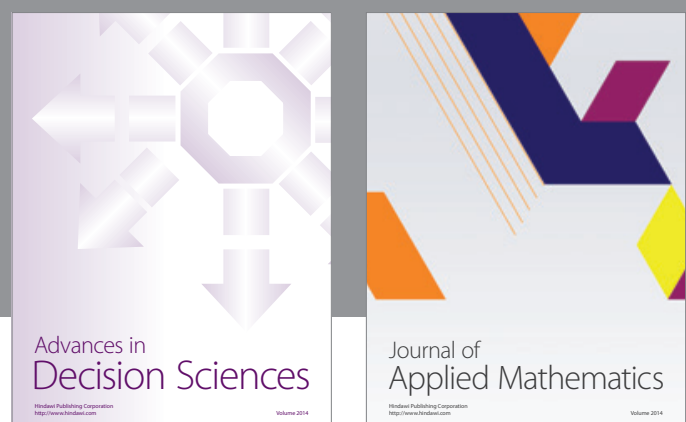

Journal of

Applied Mathematics
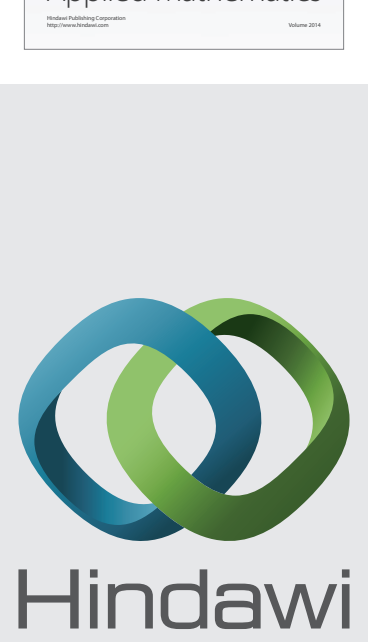

Submit your manuscripts at http://www.hindawi.com
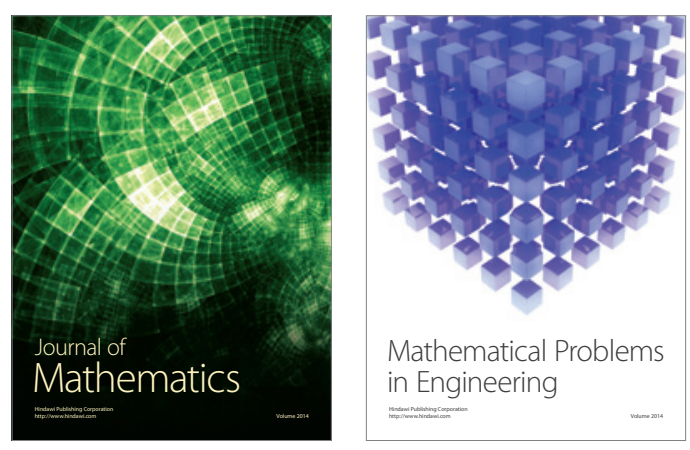

Mathematical Problems in Engineering
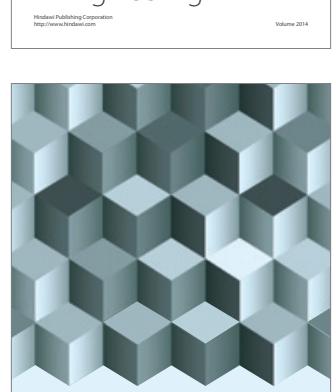

Journal of

Function Spaces
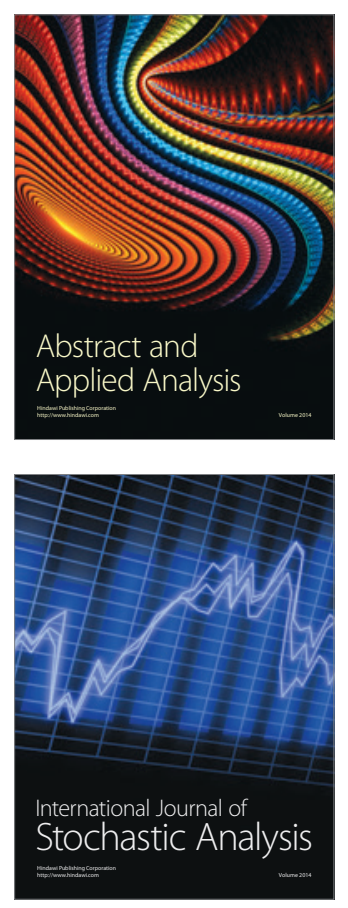

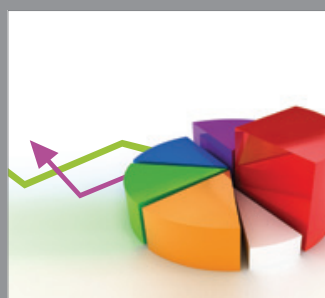

ournal of

Probability and Statistics

Promensencen
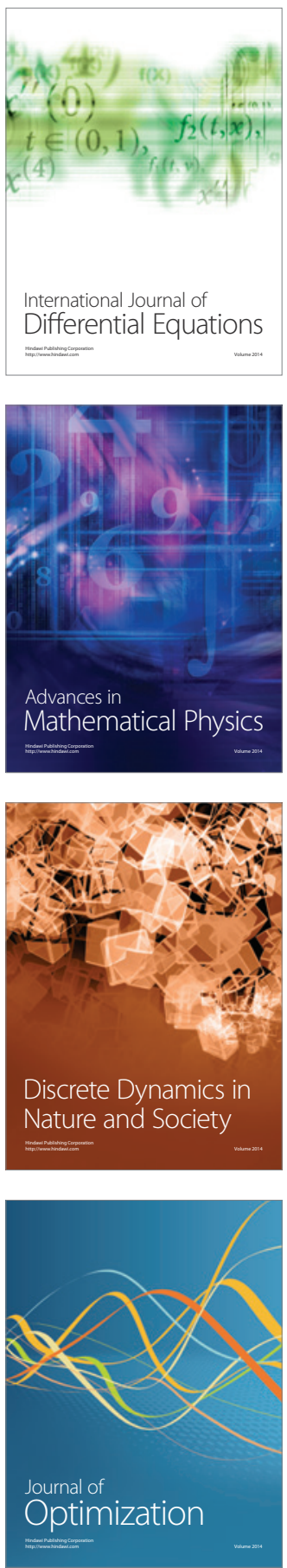